International Journal of Pure and Applied Mathematics

Volume 93 No. $6 \quad 2014,779-781$

ISSN: 1311-8080 (printed version); ISSN: 1314-3395 (on-line version)

url: http://www.ijpam.eu

doi: http://dx.doi.org/10.12732/ijpam.v93i6.2

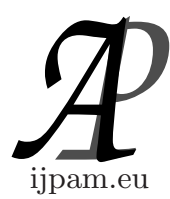

\title{
POISSON APPROXIMATION FOR INDEPENDENT NEGATIVE BINOMIAL RANDOM VARIABLES
}

\author{
K. Teerapabolarn \\ Department of Mathematics \\ Faculty of Science \\ Burapha University \\ Chonburi, 20131, THAILAND
}

\begin{abstract}
We give a bound for the total variation distance between the distribution of a sum of independent negative binomial random variables and an appropriate Poisson distribution with mean $\sum_{i=1}^{n} \frac{r_{i} q_{i}}{p_{i}}$, where $r_{i}$ and $p_{i}=1-q_{i}$ are parameters of each negative binomial distribution. It is indicated that the distribution of the sum can be approximated by the Poisson distribution with this mean when each $r_{i} q_{i}$ is small.
\end{abstract}

AMS Subject Classification: 62E17, 60F05, 60G50

Key Words: negative binomial distribution, Poisson distribution, Poisson approximation, $w$-function

\section{Introduction}

Let $X_{1}, \ldots, X_{n}$ be $n$ independently distributed negative binomial random variables, each with probability $P\left(X_{i}=k\right)=\frac{\Gamma\left(r_{i}+k\right)}{\Gamma\left(r_{i}\right) k !} q_{i}^{k} p_{i}^{r_{i}}, k \in \mathbb{N} \cup\{0\}$, mean $\mu_{i}=\frac{r_{i} q_{i}}{p_{i}}$ and variance $\sigma_{i}^{2}=\frac{r_{i} q_{i}}{p_{i}^{2}}$ where $q_{i}=1-p_{i}$. Let $\mathbf{S}_{n}=\sum_{i=1}^{n} X_{i}$ and $\mathbf{P}_{\lambda}$ denote the the Poisson random variable with mean $\lambda$. Note that, if all $r_{i} q_{i}$ are small, then the distribution of $\mathbf{S}_{n}$ is approximately a Poisson distribution with mean $\lambda$. For $\lambda=\sum_{i=1}^{n} \lambda_{i}=\sum_{i=1}^{n} r_{i} q_{i}$,Vellaisamy and Upadhye [3] gave a

Received: December 25, 2013

(C) 2014 Academic Publications, Ltd. url: www.acadpubl.eu 
bound in the form of

$$
d_{T V}\left(\mathbf{S}_{n}, \mathbf{P}_{\lambda}\right) \leq \sum_{i=1}^{n} \frac{r_{i} q_{i}^{2}}{p_{i}} \min \left\{1, \frac{1}{\sqrt{2 \lambda e}}\right\}
$$

where $d_{T V}\left(\mathbf{S}_{n}, \mathbf{P}_{\lambda}\right)=\sup _{A \subseteq \mathbb{N} \cup\{0\}}\left|P\left(\mathbf{S}_{n} \in A\right)-P\left(\mathbf{P}_{\lambda} \in A\right)\right|$ is the total variation distance between the distribution of $\mathbf{S}_{n}$ and the Poisson distribution. In this paper, we give a bound on $d_{T V}\left(\mathbf{S}_{n}, \mathbf{P}_{\lambda}\right)$ for a different Poisson mean $\lambda=\sum_{i=1}^{n} \lambda_{i}=\sum_{i=1}^{n} \frac{r_{i} q_{i}}{p_{i}}$, which is derived in Section 2. In Section 3, the conclusion of this study is presented.

\section{Result}

The following lemma is also need to prove the main result.

Lemma 2.1. For $1 \leq i \leq n$, let $w_{i}$ be the $w$-function associated with the negative binomial random variable $X_{i}$, then we have the following:

$$
w_{i}(k)=p_{i}\left(1+\frac{k}{r_{i}}\right), k \in \mathbb{N} \cup\{0\} \quad[1] .
$$

Theorem 2.1. Let $\lambda=\sum_{i=1}^{n} \lambda_{i}=\sum_{i=1}^{n} \mu_{i}$ and $\sigma^{2}=\sum_{i=1}^{n} \sigma_{i}^{2}$. Then the following inequality holds:

$$
d_{T V}\left(\mathbf{S}_{n}, \mathbf{P}_{\lambda}\right) \leq \frac{1-e^{-\lambda}}{\lambda} \sum_{i=1}^{n} \frac{r_{i} q_{i}^{2}}{p_{i}^{2}}
$$

Proof. Since $\lambda_{i}-\sigma_{i}^{2} w_{i}(k)=\frac{r_{i} q_{i}}{p_{i}}-\frac{r_{i} q_{i}}{p_{i}^{2}} p_{i}\left(1+\frac{k}{r_{i}}\right)=-\frac{k q_{i}}{p_{i}} \leq 0$ for every $k \geq 0$, it follows from [2] that

$$
\begin{aligned}
d_{T V}\left(\mathbf{S}_{n}, \mathbf{P}_{\lambda}\right) & \leq \frac{1-e^{-\lambda}}{\lambda}\left|\lambda-\sigma^{2}\right| \\
& =\frac{1-e^{-\lambda}}{\lambda}\left(\sigma^{2}-\lambda\right) \\
& =\frac{1-e^{-\lambda}}{\lambda} \sum_{i=1}^{n}\left(\frac{r_{i} q_{i}}{p_{i}^{2}}-\frac{r_{i} q_{i}}{p_{i}}\right) \\
& =\frac{1-e^{-\lambda}}{\lambda} \sum_{i=1}^{n} \frac{r_{i} q_{i}^{2}}{p_{i}^{2}}
\end{aligned}
$$


Hence (2.2) holds.

Corollary 2.1. For $r_{1}=r_{2}=\cdots=r_{n}=1$, then $\lambda=\sum_{i=1}^{n} \frac{q_{i}}{p_{1}}$ and

$$
d_{T V}\left(\mathbf{S}_{n}, \mathbf{P}_{\lambda}\right) \leq \frac{1-e^{-\lambda}}{\lambda} \sum_{i=1}^{n} \frac{q_{i}^{2}}{p_{i}^{2}} .
$$

The result (2.3) is a Poisson approximation for a sum of independent geometric random variables, which is the same result as in [2].

When all $X_{i}$ are identically distributed random variables, thus immediately from the Theorem 2.1, we have the following Corollary.

Corollary 2.2. If $r_{1}=r_{2}=\cdots=r_{n}=r$ and $p_{1}=p_{2}=\cdots=p_{n}=p$, then $\lambda=\frac{n r q}{p}$ and the following inequality holds:

$$
d_{T V}\left(\mathbf{S}_{n}, \mathbf{P}_{\lambda}\right) \leq\left(1-e^{-\lambda}\right) \frac{q}{p} .
$$

\section{Conclusion}

In this study, a bound on the total variation distance between the distribution of a sum of independent negative binomial random variables and an appropriate Poisson distribution with a different mean $\lambda$ was obtained. With this bound, it is seen that the distribution of the summands can be approximated by the Poisson distribution with mean $\sum_{i=1}^{n} \lambda_{i}=\sum_{i=1}^{n} \frac{r_{i} q_{i}}{p_{i}}$ when $r_{i} q_{i}$ is small for every $i \in\{1, \ldots, n\}$.

\section{References}

[1] R. Kun, K. Teerapabolarn, A piontwise Poisson approximation by wfunctions, Appl. Math. Sci., 6 (2012), 5029-5037.

[2] K. Teerapabolarn, An extnsion of Poisson approximation by $w$-functions, Int. J. Pure Appl. Math., 87 (2013), 529-534.

[3] P. Vellaisamy, N.S. Upadhye, Compound negative binomial approximations for sums of random variables, Prob. Math. Stat., 29 (2009), 205-226. 
